\title{
Evaluation of a Double-Shell Integrated Scanning Lens Antenna
}

\author{
Jorge R. Costa, Member, IEEE, Mário G. Silveirinha, Member, IEEE, and Carlos A. Fernandes, Senior Member, IEEE
}

\begin{abstract}
This letter presents the design and evaluation of a high dielectric constant double-material integrated lens antenna for multibeam or scanning applications. The lens is formed by two shaped embedded shells of different material permittivity to enhance both power transmission across the lens interfaces and the lens scanning characteristics over a broad frequency range, when the feeds are integrated at the lens base. Design is based on geometrical optics formulation and the lens performance is evaluated using the classic hybrid geometrical optics/physical optics approach (GO/PO). The proposed lens concept is experimentally validated with a fabricated MACOR/acrylic prototype, showing less than 1-dB scan loss up to $\pm 20^{\circ}$ with Gaussicity better than $95 \%$ over $40 \%$ frequency band at millimeter waves.
\end{abstract}

Index Terms-Integrated lens antennas, millimeter and submillimeter wave antennas, scanning antennas, shaped beam antennas.

\section{INTRODUCTION}

I NTEGRATED LENS ANTENNAS (ILAs) have been successfully used in millimeter and submillimeter wave applications [1]-[3]. A dielectric lens placed over a planar integrated antenna eliminates substrate modes and further allows producing high directivity beams. The possibility of integrating an array of compact printed feeds from a monolithic receiver directly at the base of the lens is very appealing for scanning or multibeam applications. The usual problem is beam degradation and increase of internal reflections for increasing off-axis feed positions [1], [2].

At submillimeter waves, the integrated feed is usually produced within one layer of metallization (like the slot-based printed feed) [3], [4], which results in a bidirectional radiation pattern. However, for a slot printed directly at the planar base of the lens, the fraction of power that is coupled to the lens increases with the permittivity as $\varepsilon_{r}^{3 / 2}$ [5]. A permittivity in the order of 11 is often used to enhance this fraction. But then, the high contrast at the lens air-dielectric interface originates

Manuscript received October 08, 2008. First published October 31, 2008; current version published nulldate. This work was developed in the framework of ILASH project, under European Space Agency-ESTEC Contract 17514.

J. R. Costa is with the Instituto de Telecomunicações, IST, 1049-001 Lisboa, Portugal and also with the Departamento de Ciências e Tecnologias da Informação, Instituto Superior de Ciências do Trabalho e da Empresa, 1649-026 Lisboa, Portugal (e-mail: Jorge.Costa@1x.it.pt; jorge.cesta@isete.pt).

M. G. Silveirinha is with the Electrical Engineering Department, Instituto de Telecomunicações, Departamento de Engenharia Electrotécnica, Pólo II da Universidade de Coimbra, 3030 Coimbra, Portugal (e-mail: Mario.Silveirinha@co.it.pt; mgonealo@eo.it.pt).

C. A. Fernandes is with the Instituto de Telecomunicações, Instituto Superior Técnico, 1049-001 Lisboa, Portugal (e-mail: Carlos.Fernandes@1x.it.pt).

Digital Object Identifier 10.1109/LAWP.2008.2008403 significant internal reflection, especially for off-axis feed positions. A $\lambda / 4$ matching layer may be used to overcome this limitation but that is a frequency selective solution; it is far from pairing for instance with the 1:2.5 bandwidth of the integrated feed presented in [4]. It should be noted that the need for uniplanar printed feeds is incompatible with either classic single material two-surface scanning lenses [6], [7] or with the two-shell spherical lens studied in [8] (which has a perfect scanning characteristic due to its spherical symmetry), since in these configurations the feed is located well outside the lens, in air.

The scarce integrated lens configurations reported in the literature for scanning or multibeam applications are based only on extended hemispherical or elliptical lens configurations [1]-[3]. In these canonical structures, the lens shape is fixed, and thus the only available degree of freedom for design is the lens extension length, which determines the feed distance to the lens radiating surface.

In this letter, we propose a novel integrated lens configuration for multibeam or scanning applications, formed by two embedded shaped shells. Unlike the configuration studied in [8], our proposal enables the use of uniplanar printed feeds. It will be shown that because the shape of the shells is obtained from a geometrical optics (GO) formulation and because the resulting shell thickness does not verify any resonant condition, the power transmission through the shaped double-shell lens (SDSL) is to a large extent independent of frequency. Furthermore, due to the design flexibility offered by the two-shell configuration, the permittivity contrast at the two dielectric interfaces can be chosen significantly lower than in a single material lens, thus favoring the reduction of internal reflections. Another consequence of a GO-based lens design is that the scan angle dependence with the feed off-axis position will be practically independent of frequency, as will be confirmed ahead with physical optics (PO) analysis and measurements. This makes the integrated SDSL quite appealing for multifrequency or ultrawideband applications. The challenge for this high-permittivity integrated lens is to minimize internal reflection loss over an extended frequency bandwidth (at least over $40 \%$ bandwidth) and to ensure a stable main beam shape in the same band independent of the scanning angle.

\section{LENS DESIGN}

Consider the geometry of Fig. 1. The structure is axial-symmetric, formed by two embedded shells of different materials; the inner shell presents the higher permittivity value $\varepsilon_{r 2}$ and the outer shell the lower value $\varepsilon_{r 1}$. An array of feeds is distributed 


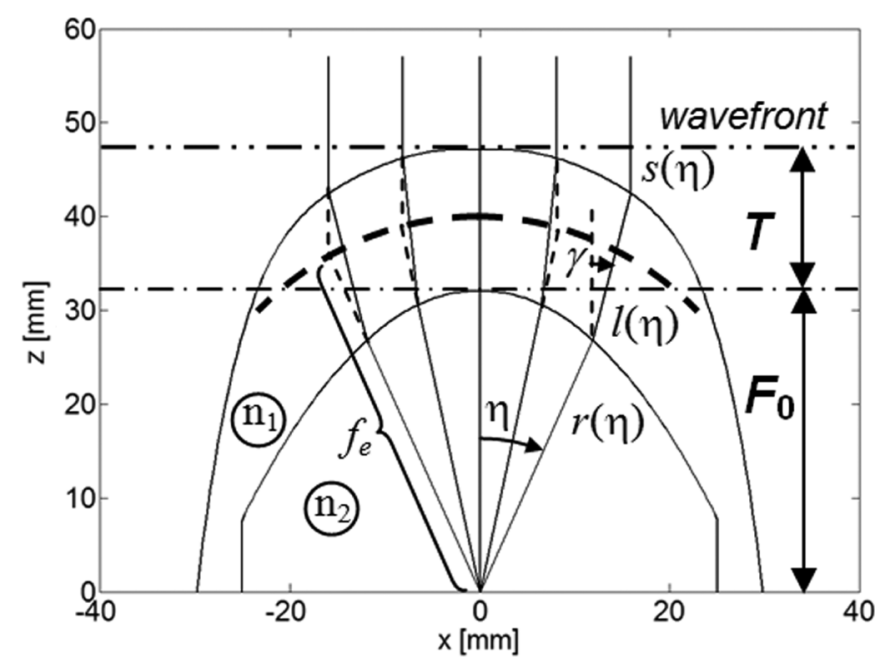

Fig. 1. Geometry of the SDSL lens.

at the planar base of the lens within a small area centered with the lens axis.

The possibility to shape two lens surfaces enables that two independent design goals can be specified (in a single-material lens, only one design goal can be defined):

1) a beam collimation condition, i.e., the output rays emerging from the lens should be parallel to each other;

2) a scanning condition that ensures reduced aberration of the output beam.

We define the inner lens surface by the (unknown) function $r(\eta)$ and the outer lens surface by the (unknown) length $l(\eta)$ and angle $\gamma(\eta)$ (see Fig. 1). The lens axial thickness is $F_{0}$ and $T$ for the inner and outer shells, respectively.

It is well known that a lens satisfying the so called Abbe sine condition is free from comma aberration for a small off-axis transversal displacement of the feed [9]. The Abbe sine condition is verified when the intersection points of the extended $r(\eta)$ rays departing from the on-axis feed and the corresponding extended transmitted $s(\eta)$ rays all lie over an arc of circumference with a certain radius $f_{e}$ centered at the sensor [9]. This is represented by the thick dashed arc in Fig. 1. In view of the geometry of Fig. 1, the Abbe sine condition can be written as

$$
\left(f_{e}-r\right) \sin (\eta)=l \sin (\gamma)
$$

Snell's law at the inner interface implies that

$$
\frac{d r}{d \eta}=\frac{\left(n_{1} / n_{2}\right) r \sin (\eta-\gamma)}{\left(n_{1} / n_{2}\right) \cos (\eta-\gamma)-1}
$$

where $n_{1}=\sqrt{\varepsilon_{r 1}}$ and $n_{2}=\sqrt{\varepsilon_{r 2}}$. In order that the optical path length of every ray is the same at the exiting wavefront, it is required that

$$
\begin{aligned}
n_{2} r+n_{1} l+s & =n_{2} F_{0}+n_{1} T \\
s & =F_{0}+T-r \cos (\eta)-l \cos (\gamma) .
\end{aligned}
$$

Equations (1)-(3) can be solved simultaneously to determine both the inner and the outer shell profiles, taking $\eta$ as the independent variable. The initial condition for $\eta=0$ is $r=F_{0}, l=$ $T$, and $\gamma=0$ and the integration is extended up to $\eta=\eta_{\text {edge }}$, where generally $\eta_{\text {edge }}>\pi / 2$. The calculation is stopped at the point where the outer shell intersects the $f_{e}$ circle or where the total internal reflection condition is reached.

For a given combination of $n_{1}, n_{2}, F_{0}$, and $T$ values, the $f_{e}$ parameter can be adjusted between $F_{0}$ and $F_{0}+T$ to control the shape of the lens surfaces (and indirectly the lens scanning characteristics). The lens shape obtained with the above GO-based formulation is independent of the absolute dimensions, and thus, for instance $F_{0}$, can be taken as a scaling factor.

It is noteworthy that, in line with the classical Abbe formulation for single material lenses [6], the above design equations consider only the central feed. The imposed Abbe condition implicitly determines the scanning behavior for off-axis feeds. The described formulation is implemented in the ILASH [AU: Please define ILASH $]_{\text {lens design and opti- }}$ mization tool [10], which was used to design the lens.

\section{PO ANALYSIS AND EXPERIMENTAL RESULTS}

\section{A. Considerations About Dielectric Materials}

An appropriate combination of materials for SDSL applications at submillimeter wave is silicon/fused quartz. These are low-loss materials with permittivity around 11.7/3.8. Due to limitations of our measuring equipment, the prototype had to be tested instead at millimeter waves, in the $40-70-\mathrm{GHz}$ range. However, the resulting size of the lens at these lower frequencies (60-mm diameter) makes it very difficult to machine the very hard silicon and fused quartz materials. Due to this reason, we have considered other materials compatible with milling fabrication technique. Factors such as the permittivity value, homogeneity, isotropy, and availability in large blocks were also decisive for the choice of the materials. A ceramic-based dielectric material-MACOR - with $\varepsilon_{r 2}=5.5(1-j 0.0118)$ was chosen for the inner lens and acrylic $\varepsilon_{r 1}=2.53(1-j 0.0118)$ for the outer shell. The complex permittivity was measured in the $40-45 \Xi \mathrm{GHz}$ and $60-65-\mathrm{GHz}$ range using a waveguide method, with uncertainty less than $0.5 \%$ for the real part of the permittivity. The adopted square root relation between the permittivity of the inner and outer shell materials maximizes the power transfer across the double-shell lens [11]. Even though the dissipation loss of these alternative materials is relatively high, they enable to effectively demonstrate the main characteristics of the SDSL, as discussed ahead.

\section{B. Lens Evaluation}

The selected parameter values for SDSL evaluation are $F_{0}=$ $32.02 \mathrm{~mm}, T=15 \mathrm{~mm}$, and $f_{e}=39.14 \mathrm{~mm}$. The corresponding lens profile is shown in Fig. 1. The profile calculation stops at $\eta_{\text {edge }}=41.6^{\circ}$ and it is empirically extended down to the lens base to close the volume. This extension is shaped to avoid total internal reflection or that rays emerging from this section of the lens perturb the main lobe. The lens diameter is $D=60 \mathrm{~mm}(\sim$ $\left.13.5 \lambda_{0}\right)$ at the base. These dimensions are intended to produce $25-28-\mathrm{dBi}$ directivity at the test frequency range. There is no basic limitation in the method or the scope of the results, and the described lens can be scaled or redesigned for other frequency range or other material parameters. 

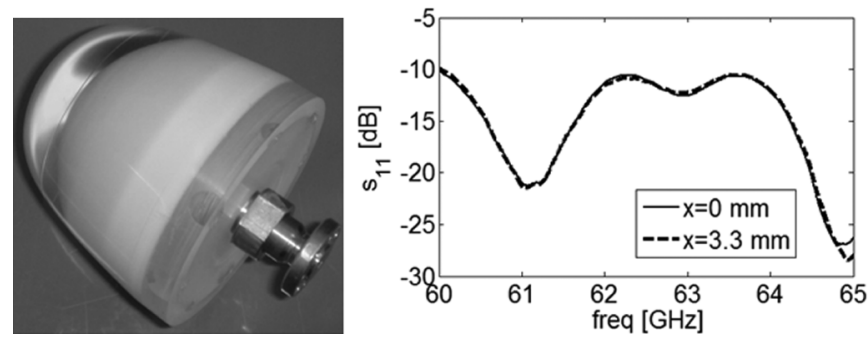

Fig. 2. (a) Fabricated MACOR/acrylic lens prototype, showing the attached 60-GHz band waveguide feed. (b) Measured return loss at the waveguide input port, when radiating into the lens prototype, for two feed positions.

Fig. 2(a) shows the fabricated lens prototype. The milling technique was used to produce the two lens shells and an intentional $0.1-\mathrm{mm}$ gap between them was filled with glue of the same dielectric constant as acrylic. Two identical tapered dielectric-filled $\left(\varepsilon_{r}=8.8\right.$ and $\varepsilon_{r}=11.1$ ) $\mathrm{TE}_{10}$ waveguide feeds (one for $43 \mathrm{GHz}$ with $2 \mathrm{~mm} \times 2 \mathrm{~mm}$ aperture and another for 62.5 $\mathrm{GHz}$ with $1.4 \mathrm{~mm} \times 1.4 \mathrm{~mm}$ aperture) were designed and fabricated to produce exactly the same radiation pattern at the two frequencies, with linear polarization, similar to that of an appropriate printed antenna. This enables to test the broadband behavior of the lens by sampling its response at two well separated frequencies as if a single broadband feed with constant radiation pattern were used. In both bands, the feeds radiation pattern is almost rotationally symmetric with 7.4-dBi directivity and reduced radiation level towards the lens extrapolated regions near the base. These dedicated waveguide feeds enable quick assembly/disassembly at the lens base and its precise alignment for repeatable and reliable measurements and very pure linear polarization. Appropriate broadband printed feeds with integral mixer have been developed and described elsewhere [4], but their application to the SDSL falls outside the scope of this letter.

Fig. 2(b) shows the measured return loss at the waveguide port in the 60-GHz band, when it is attached to the base of the SDSL for on-axis and off-axis feeding. The curves show negligible dependence with feed translation, an indirect indication of the low level of internal reflection rays that hit back on the feed aperture. Similar type of curves is obtained for the $400 \mathrm{GHz}$ feed.

Figs. 3 and 4 show the measured SDSL radiation patterns at 62.5 and $43 \mathrm{GHz}$, respectively, for feed positions $x=0,1.1$, 2.2 , and $3.3 \mathrm{~mm}$ and $y=2.02 \mathrm{~mm}$. Measured results (thick lines) are superimposed on simulations (dotted lines) obtained from a hybrid GO/PO analysis (ILASH tool [10]) considering only first-order internal reflections. Simulation results are also shown for the feed positions $x=4.4$ and $5.5 \mathrm{~mm}$.

In all the tested cases, the agreement between the measured radiation pattern and the corresponding $\mathrm{GO} / \mathrm{PO}$ simulated results is good in terms of beam shape versus scan angle, sidelobe level, and beam direction. GO/PO produces a good prediction for the SDSL in part because internal reflections are quite low (quantification is given ahead). This is due to the moderate contrast at both lens interfaces and due to the favorable angles of ray incidence at the lens interfaces, comfortably away from the critical angle that originates total internal reflection. This fact also
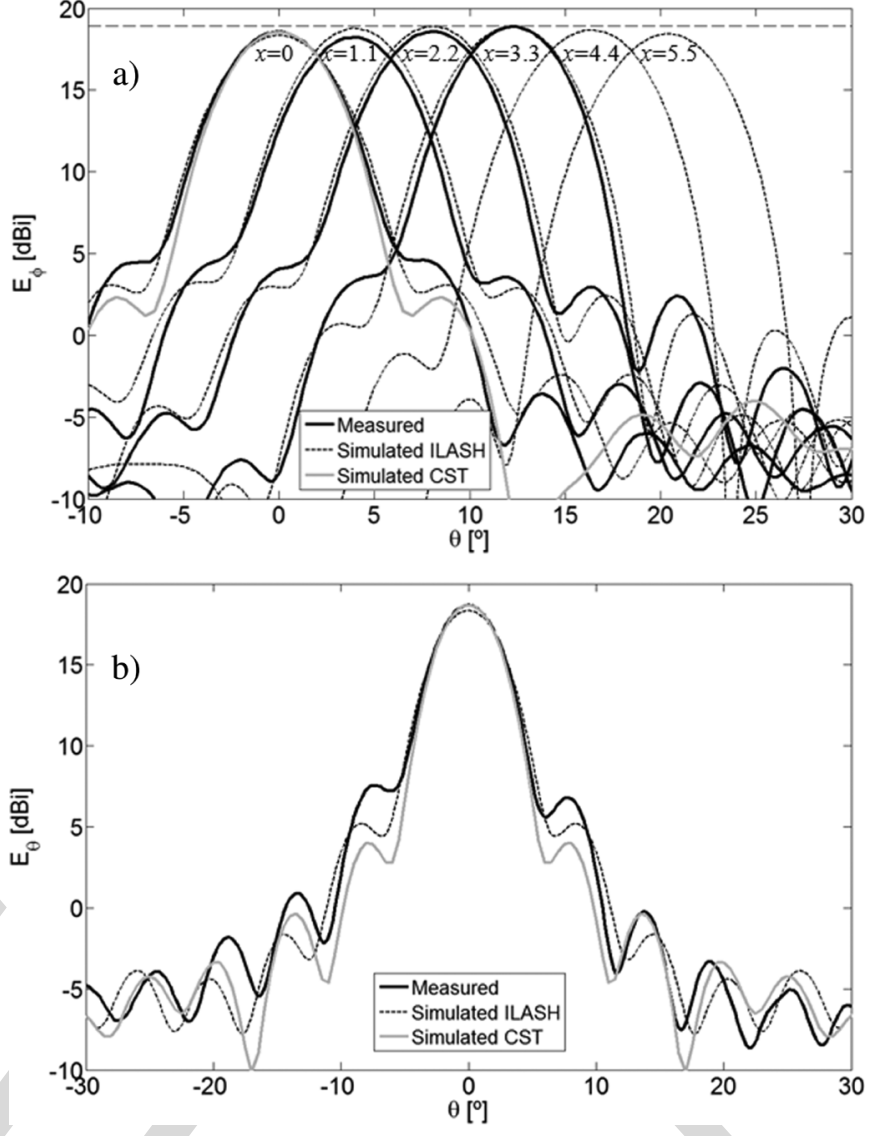

Fig. 3. Simulated and measured radiation patterns at $62.5 \mathrm{GHz}$, leveled to max gain. CST curve is downshifted. (a) H-plane, with feed at $x=0,1.1,2.2,3.3$, 4.4, and $5.5 \mathrm{~mm}$. (b) E-plane with feed at $x=0$.

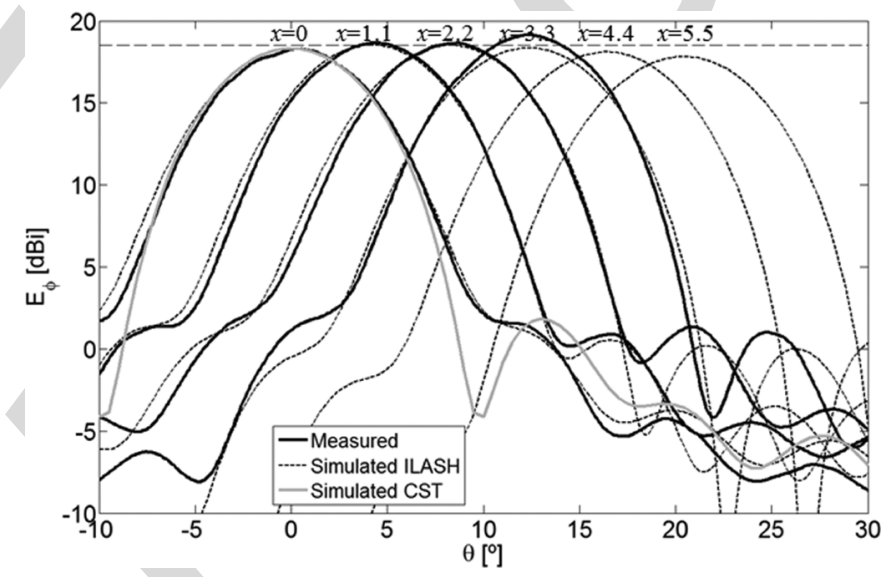

Fig. 4. Simulated and measured radiation patterns at $43 \mathrm{GHz}$, leveled to max gain: H-plane, with feed at $x=0,1.1,2.2,3.3,4.4$, and $5.5 \mathrm{~mm}$.

minimizes the excitation of the "lateral wave" that is present in other collimated beam lenses such as the elliptical lens [12] and cannot be accounted for with GO/PO analysis. These benefits result from using two shaped refraction surfaces.

Figs. 3 and 4 show that the scan angle dependence with feed off-axis position is linear. The main beam preserves its shape with scanning angle up to $20^{\circ}$, while the gain scan loss is less than $1 \mathrm{~dB}$ (see Table I). Beam degradation is quantified through 
TABLE I

Calculated and Measured Lens Performance Indicators for SiX DIFFERENT FEED OFF-AXIS POSITIONS AND FOR TWO FREQUENCIES WITH $40 \%$ SPAN

\begin{tabular}{|c|c|c|c|c|c|c|c|c|}
\hline & & \multicolumn{6}{|c|}{ Simulation ILASH } & \multirow{2}{*}{$\begin{array}{c}\text { Meas. } \\
G \\
{[\mathrm{dBi}]}\end{array}$} \\
\hline & $\begin{array}{c}x \\
{[\mathrm{~mm}]}\end{array}$ & $\xi\left[{ }^{\circ}\right]$ & $\begin{array}{c}L_{\mathrm{r}} \\
{[\mathrm{dB}]}\end{array}$ & $\begin{array}{c}L_{\mathrm{d}} \\
{[\mathrm{dB}}\end{array}$ & $\begin{array}{l}L_{\mathrm{WG}} \\
{[\mathrm{dB}}\end{array}$ & $\begin{array}{c}D \\
{[\mathrm{dBi}]}\end{array}$ & $\begin{array}{c}G \\
{[\mathrm{dBi}]}\end{array}$ & \\
\hline \multirow{6}{*}{ 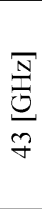 } & 0.0 & 0.0 & 0.7 & 3.7 & 2.2 & 24.9 & 18.3 & 18.3 \\
\hline & 1.1 & 4.0 & 0.5 & 3.7 & 2.2 & 25.0 & 18.6 & 18.7 \\
\hline & 2.2 & 8.0 & 0.6 & 3.7 & 2.2 & 25.0 & 18.5 & 18.6 \\
\hline & 3.3 & 12.1 & 0.7 & 3.7 & 2.2 & 25.0 & 18.4 & 19.1 \\
\hline & 4.4 & 16.1 & 0.9 & 3.7 & 2.2 & 24.9 & 18.1 & --- \\
\hline & 5.5 & 20.2 & 1.1 & 3.7 & 2.2 & 24.9 & 17.9 & --- \\
\hline \multirow{6}{*}{ 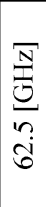 } & 0.0 & 0.0 & 0.6 & 5.6 & 3.2 & 27.7 & 18.3 & 18.6 \\
\hline & 1.1 & 3.9 & 0.5 & 5.5 & 3.2 & 28.0 & 18.8 & 18.2 \\
\hline & 2.2 & 7.9 & 0.5 & 5.6 & 3.2 & 28.2 & 18.9 & 18.6 \\
\hline & 3.3 & 11.9 & 0.7 & 5.5 & 3.2 & 28.3 & 18.9 & 18.9 \\
\hline & 4.4 & 16.1 & 0.9 & 5.6 & 3.2 & 28.3 & 18.6 & --- \\
\hline & 5.5 & 20.1 & 1.2 & 5.5 & 3.2 & 28.3 & 18.4 & --- \\
\hline
\end{tabular}

the Gaussicity parameter, which measures the degree of coupling between the lens far-field radiation pattern intensity and a fundamental Gaussian-beam [3]. The Gaussicity value for the main beam changes with feed off-axis position from $99.3 \%$ to $95.5 \%$. In this calculation, the main beam is identified with the angular interval where the radiation intensity is over $-18 \mathrm{~dB}$ for $43 \mathrm{GHz}$ and $-15 \mathrm{~dB}$ for $62.5 \mathrm{GHz}$. Maximum Gaussicity is achieved when coupling to a Gaussian beam with $-10 \mathrm{~dB}$ full-beamwidth $\theta_{\max }=15.02^{\circ}$ at $43 \mathrm{GHz}$ and $\theta_{\max }=10.08^{\circ}$ at $62.5 \mathrm{GHz}$.

Table I also presents the loss partial terms, obtained from the GO/PO analysis. First-order internal reflections loss $L_{r}$ for both frequency bands is less than $1 \mathrm{~dB}$ and its variation with off-axis feed position is less than $0.6 \mathrm{~dB}$. This is a quite promising characteristic for an integrated scanning lens. MACOR and Plexiglas high dissipation losses $L_{d}$ and feed internal losses $L_{W G}$ excessively penalize the lens output gain $G$, but these can be overcome by using lower loss materials and an appropriate printed feed. For silicon/fused quartz, $L_{d}$ would be 0.3 and $0.5 \mathrm{~dB}$ for 43 and $62.5 \mathrm{GHz}$, respectively. Dissipation losses do not change the lens design. One issue that might be raised is that in a low-loss SDSL design the internally reflected rays are not damped, and this might corrupt the characteristics of the lens. Full wave simulations [finite integration method (FIM)] obtained using CST Microwave Studio show that this is not the case. Results for the same MACOR/acrylic lens assuming zero dissipation losses and on-axis feed position are depicted in Figs. 3 and 4 (dashed lines); the gain is higher in this case, but the CST curves were rescaled for better comparison. Despite the limited available computer resources for accurate CST modeling of the SDSL (6-GB RAM were used corresponding to a mesh of 16 millions cells), the radiation pattern of the ideal lossless lens is similar with that of the measured lens. This demonstrates that the effect of internal reflections, even in the absence of dissipation loss, is relatively low. This property stems from the relatively low dielectric contrast between the different materials, which was made possible by the double shell design.

It is important to note that the lens scanning performance in terms of beam direction, scan loss, and Gaussicity remains prac- tically unchanged between 43.5 and $62.5 \mathrm{GHz}$, a $40 \%$ span. Of course, directivity changes with frequency in the same manner as in any collimated beam lens, including the spherical lens from [8]. This can be tackled with appropriate feed design; this specific aspect of the design falls outside the scope of this letter and will be presented elsewhere.

\section{CONCLUSION}

This letter presents a very promising lens solution for direct mount on top of a monolithic integrated feed circuit at millimeter or submillimeter waves in scanning or multibeam applications. The high-permittivity double-shell lens concept conciliates efficient power coupling from uniplanar feeds and reduced lens internal reflections over large bandwidths in excess of $40 \%$. It offers in that band less than $1-\mathrm{dB}$ gain scan loss over $+/-20^{\circ}$ angular interval, less than 1-dB reflection losses, and better than $95 \%$ Gaussicity. Directivity is $25-28 \mathrm{dBi}$ for $43-62 \mathrm{GHz}$.

Although the materials used for lens prototyping present relatively high loss tangent, it was demonstrated using a full wave FIM simulator that dissipation losses do not affect significantly the main characteristics of the SDSL (apart from the gain). Usual low-loss materials such as silicon and fused quartz are appropriate for fabrication of submillimeter wave lenses. The $\mathrm{GO} / \mathrm{PO}$-based analysis tool is accurate enough for a correct assessment of the SDSL performance. Since the computation is very fast, this is the indicated approach for optimization loops, which would be impracticable with full wave tools. Lens shape optimization is the next step to enhance SDSL performance.

\section{REFERENCES}

[1] D. Filipovic, G. Gauthier, S. Raman, and G. Rebeiz, "Off-axis properties of silicon and quartz dielectric lens antennas," IEEE Trans. Antennas Propag., vol. 45, no. 5, pp. 760-766, May 1997.

[2] X. Wu, G. Eleftheriades, and T. Van Deventer-Perkins, "Design and characterization of single- and multiple-beam mm-wave circularly polarized substrate lens antennas for wireless communications," IEEE Trans. Microw. Theory Tech., vol. 49, no. 3, pp. 431-441, Mar. 2001.

[3] D. Filipovic, S. Gearhart, and G. Rebeiz, "Double-slot antennas on extended hemispherical and elliptical silicon dielectric lenses," IEEE Trans. Microw. Theory Tech., vol. 41, no. 10, pp. 1738-1749, Oct. 1993.

[4] J. Costa and C. Fernandes, "Broadband slot feed for integrated lens antennas," IEEE Antennas Wireless Propag. Lett., vol. 6, pp. 396-400.

[5] D. Rutledge, D. Neikirk, and D. Kasilingam, , K. Button, Ed., "Integrated circuit antennas," in Infrared and Millimeter-Waves. New York: Academic, 1983, vol. 10 nn 1-90.

[6] J. Brown, Microwave Lenses. London, U.K.: Wiley, 1953.

[7] A. Peebles, "A dielectric bifocal lens for multibeam antenna applications," IEEE Trans. Antennas Propag., vol. AP-36, no. 5, pp. 599-606, May 1988.

[8] H. Mosallaei and Y. Rahmat-Samii, "Nonuniform Luneburg and twoshell lens antennas: Radiation characteristics and design optimization," IEEE Trans. Antennas Propag., vol. 49, no 1. pp. 60-69. Jan. 2001.

[9] M. Born and E. Wolf, Principles of Optids. New York:Pergamon,

[10] [ÄU: Please provide page range and define APS/URSI] E. Lima, J. Costa, M. Silveirinha, and C. Fernandes, "ILASH-Software tool for the design of integrated lens antennas," in Proc. IEEE APS/URSI Symp., San Diego, CA,JJul. 2008.

[11] M. Silveirinha and C. Fernandes, "Shaped double-shell dielectric lenses for wireless mm-wave communications," in Proc. IEEE APS/URSI Symp., Salt Lake City, UT, Jul. 2000, pp. 1674-1677.

[12] D. Pasqualini and S. Maci, "High-frequency analysis of integrated dielectric lens antennas," IEEE Trans. Antennas Propag., vol. 52, no. 3, pp. 40-847, Mar. 2004. 